\title{
Damage control resuscitation initiated in the prehospital and transport setting: A systems approach to increasing access to blood transfusion
}

\author{
Alix J.E. Carter, MD, MPH${ }^{*}$; Russell D. MacDonald, MD, $\mathrm{MPH}^{\dagger}$
}

A life-threatening hemorrhage is the leading cause of preventable death in patients with multisystem traumatic injuries, obstetric complications, gastrointestinal bleeding, and those experiencing intraoperative and postoperative complications. ${ }^{1,2}$ Patients can deteriorate in the transport setting, prior to arrival at definitive care. While the goal is rapid transport for damage control interventions, transport may be delayed or prolonged due to patient access at the scene, limited therapeutic options at the sending/community hospital or in transport, and long distances to definitive care. The care provided during transport is an important factor in patient outcome, as is the integration with other providers to create a system of care.

In this issue of $C 7 E M$, Krook et al. describe the development of a novel program to provide packed red blood cells (pRBCs) to patients during helicopter transport to definitive care from either the location of the injury or from the community hospital. The authors describe their solution to the operational challenges in making blood products available to a large region, while balancing increased accessibility with efficient and judicious use of a scarce resource. They describe a system of rotor-wing aircraft at six locations in Western Canada. The transport program uses portable coolers to store and transport pRBCs, requiring product exchanges at 72-96 hour intervals with their partnering blood banks. The authors describe the administration of 463 units of pRBCs to 274 patients, representing approximately $3.6 \%$ of the patients transported. This percentage is comparable with other Canadian air medical transport programs (R. MacDonald, unpublished data). While all units were type $\mathrm{O}$, there are no data on what proportion was Rh-negative; given the chronic short supply of O-Rh-negative blood, its use should be limited to patients who are O-negative or patients with unknown blood group who are female and of child-bearing age. ${ }^{3}$ Data regarding the number of units dispensed are only available for one of six bases, with 898 units of pRBCs dispensed, 131 (14.6\%) of which were transfused to 81 patients, $756(84.2 \%)$ returned to the hospital, and 11 $(1.2 \%)$ wasted - an important figure to track.

The system described here has implemented a systems-based solution to previous barriers such as large start-up costs associated with more permanent installations to stock product at each air base, the outdating of unused blood product, and complicated or cumbersome cooling mechanisms. Negatives include the ongoing costs of exchanging product every 3-4 days; the need for frequent exchange of a small supply also risks not having blood product available when the existing supply is used. This paper is an important illustration of a regionalized and integrated approach to fair allocation and access to scarce resources, such as blood products and specialty services. Given the relative scarcity of blood products, their sporadic and unpredictable need and the desire to prevent wastage, bundling blood with a means of rapid transport to definitive care is an ideal way to meet patient needs while conserving a precious resource.

Given that the peak incidence of hemorrhagic deaths is within 3 hours after injury, early resuscitation with appropriate fluids in combination with strategies to control blood loss makes intuitive sense and can be life-

From the *Department of Emergency Medicine, Dalhousie University, Halifax, NS; and the tDepartment of Medicine, Emergency Medicine Fellowship Program, University of Toronto, Toronto, ON.

Correspondence to: Dr. Alix J.E. Carter, Department of Emergency Medicine, Dalhousie University, 1796 Summer St., Suite 3021, Halifax, NS B3H 3A7; Email: Alix.carter@novascotia.ca

(c) Canadian Association of Emergency Physicians

CJEM 2019;21(3):318-320

DOI 10.1017/cem.2019.28 
saving. ${ }^{4,5}$ The use of prehospital crystalloid and colloid resuscitation strategies has not been shown to improve survival, with a major prehospital clinical trial stopping early due to possible harm to patients who did not receive blood. ${ }^{6}$ Using blood products to resuscitate patients in hemorrhagic shock is not new. Prehospital plasma was used in World War II but proved inadequate in the absence of other blood components. ${ }^{7}$ Evidence for optimum hospital-based transfusion protocols for hemorrhagic shock and massive transfusions is now well-established, with minimization of crystalloid solutions and early transfusion of plasma, platelets, and red blood cells in balanced ratios. ${ }^{4}$ This approach leads to a reduction in early trauma deaths and mortality related to blood loss.

These early interventions are relevant in the air medical evacuation of patients with life-threatening hemorrhage, where transport times can be prolonged and patients may be cared for in resource-challenged settings where access to blood products is limited or non-existent. Blood products administered in to combat casualties during helicopter transport showed a survival benefit, and a recently published trial demonstrated the benefit of blood products in civilian patients with severe injuries. ${ }^{8,9}$ This approach improves outcome in patients who survive to reach the trauma centre, suggesting that damage control resuscitation is possible in the transport setting. ${ }^{7}$

Although the focus is on operational and logistical aspects, the authors provide only limited patient-centred data. There are limited or no data on crew composition or expertise, measures of transfusion safety, measurement of hemoglobin or coagulation status, hospital interventions, or mortality. As is the case with Krook et al., patients transported by crews who are specifically trained and routinely work in the transport setting have fewer adverse events and better outcomes compared with similar providers who work predominantly in the hospital setting. ${ }^{10}$ Consider also, the transfusion of pRBCs alone may not result in optimal outcome when compared with balanced-ratio resuscitation. The balanced approach has improved outcomes in patients who survive transport to the trauma centre, strongly suggesting that damage-control resuscitation should be started during transport. ${ }^{11}$ It is safe to assume that the infrastructure described to make pRBCs available in transport could accommodate plasma or other blood products, providing more optimal management of patients with hemorrhagic shock. ${ }^{9}$ The authors also indicate the availability of adjuncts for hemorrhage control, but a description of an all-encompassing prehospital protocol to address massive hemorrhage would benefit the reader.

This article illustrates the value of an integrated regional or provincial health system of care. Shortened time to definitive care improves outcomes, and activation of a transport service for a patient with hemorrhagic shock should be a key component to any regional or provincial massive hemorrhage protocol. ${ }^{11}$ Challenges of distance, geography, and local resources including blood product will persist and must be addressed. Most effectively, challenges like these will be addressed through an integrated regional strategy. Regionalization has been demonstrated to save lives in patients with multisystem traumatic injuries, STEMI, and stroke; the concept of integration to maximize access and efficiency of delivery of care is not new. ${ }^{12}$

The experience of one Canadian air medical service should motivate air medical transport services, regional referral centres, and blood supply agencies to partner and implement programs that extend the reach of damage control resuscitation methods into the prehospital and transport medicine setting. Although limited, the authors demonstrate that an air medical service can enhance their potentially life-saving capabilities, become better integrated into a regional approach to management of massive hemorrhage, and address a gap in resource-challenged settings.

Keywords: Critical care, emergency medical services, trauma

Competing interests: None declared.

\section{REFERENCES}

1. Davis JS, Satahoo SS, Butler FK, et al. An analysis of prehospital deaths: who can we save? $\mathcal{F}$ Trauma Acute Care Surg 2014;77(2):213-8.

2. Bruun MT, Pendry K, Georgsen J, et al. Patient blood management in Europe: surveys on top indications for red blood cell use and patient blood management organization and activities in seven European university hospitals. Vox Sang 2016;111(4):391-8.

3. British Committee for Standards in Haematology, Stainsby D, MacLennan S, Thomas D, Isaac J, Hamilton PJ. Guidelines on the management of massive blood loss. Br 7 Haematol 2006;135(5):634-41.

4. Holcomb JB, Tilley BC, Baraniuk S, et al. Transfusion of plasma, platelets, and red blood cells in a 1:1:1 vs a 1:1:2 ratio and mortality in patients with severe trauma: the PROPPR randomized clinical trial. $7 A M A$ 2015;313(5):471-82. 
5. Davis BL, Martin MJ, Schreiber M. Military resuscitation: lessons from recent battlefield experience. Curr Trauma Rep 2017;3:156-63.

6. Bulger EM, May S, Kerby JD, et al. Out-of-hospital hypertonic resuscitation after traumatic hypovolemic shock: a randomized, placebo controlled trial. Ann Surg 2011; 253:431-41.

7. Cannon JW, Fischer JE.Edward D. Churchill as a combat consultant: lessons for the senior visiting surgeons and today's military medical corps. Ann Surg 2010;251:566-72.

8. Shackelford SA, Del Junco DJ, Powell-Dunford N, et al. Association of prehospital blood product transfusion during medical evacuation of combat casualties in Afghanistan with acute and 30-day survival. 7AMA 2017;318:1581-91.

9. Sperry JL, Guyette FX, Brown JB, et al. Prehospital plasma during air medical transport in trauma patients at risk for hemorrhagic shock. N Engl 7 Med 2018;379:315-26.
10. Vos GD, Nissen AC, Nieman FH, et al. Comparison of interhospital pediatric intensive care transport accompanied by a referring specialist or a specialist retrieval team. Intensive Care Med 2004;30(2):302-8.

11. Cannon JW, Khan MA, Raja AS, et al. Damage control resuscitation in patients with severe traumatic hemorrhage: a practice management guideline from the Eastern Association for the Surgery of Trauma. 7 Trauma Acute Care Surg 2017;82:605-17.

12. Sampalis JS, Denis R, Lavoie A, et al. Trauma Care Regionalization: A Process-Outcome Evaluation. 7 Trauma 1999;46 (4):565-79.

13. Krook C, O'Dochartaigh D, Martin D, Piggott Z, Deedo R, Painter S, van Werkhoven G, McKay D, et al. Blood on board: The development of a prehospital blood transfusion program in a Canadian helicopter emergency medical service. CFEM 2019;21(3):365-373. 